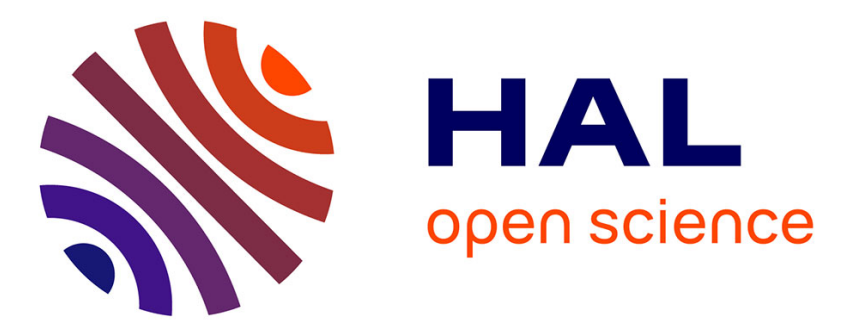

\title{
Potential input from metabolomics for exploring and understanding the links between environment and health.
}

Nathalie Bonvallot, Marie Tremblay-Franco, Cecile Chevrier, Cécile Canlet, Laurent Debrauwer, Jean Pierre J. P. Cravedi, Sylvaine Cordier

\section{To cite this version:}

Nathalie Bonvallot, Marie Tremblay-Franco, Cecile Chevrier, Cécile Canlet, Laurent Debrauwer, et al.. Potential input from metabolomics for exploring and understanding the links between environment and health.. Journal of Toxicology and Environmental Health, Part B: Critical Reviews, 2014, 17 (1), pp.21-44. 10.1080/10937404.2013.860318 . hal-00976116

\section{HAL Id: hal-00976116 \\ https://hal-univ-rennes1.archives-ouvertes.fr/hal-00976116}

Submitted on 18 Jul 2014

HAL is a multi-disciplinary open access archive for the deposit and dissemination of scientific research documents, whether they are published or not. The documents may come from teaching and research institutions in France or abroad, or from public or private research centers.
L'archive ouverte pluridisciplinaire HAL, est destinée au dépôt et à la diffusion de documents scientifiques de niveau recherche, publiés ou non, émanant des établissements d'enseignement et de recherche français ou étrangers, des laboratoires publics ou privés. 
Potential input from metabolomics for exploring and understanding the links between environment and health

BONVALLOT NATHALIE (1,2,3), TREMBLAY-FRANCO MARIE (3), CHEVRIER CECILE (1,4), CANLET CECILE (3), DEBRAUWER LAURENT (3), CRAVEDI JEANPIERRE (3), CORDIER SYLVAINE $(1,4)$

(1) INSERM UMR1085 IRSET, Rennes, France

(2) EHESP Rennes, Sorbonne Paris Cité, France

(3) INRA UMR 1331Toxalim; University of Toulouse, INP, ENVT, EIP, UPS, UMR1331, Toulouse, France

(4) University of Rennes I, Rennes, France

\section{Corresponding author}

Nathalie Bonvallot

EHESP School of Public Health, Avenue du Professeur Léon Bernard, CS74312, F-35043

Rennes Cedex, France. Tel: +332 990226 22. Fax: +332 990226 75. E-mail: nathalie.bonvallot@ehesp.fr

\section{Other authors}

Marie Tremblay-Franco, INRA - UMR 1331 TOXALIM, Bâtiment A, 180 chemin de tournefeuille, BP 93173, 31027 Toulouse cedex 3, France. Tel : +335 612857 13. Fax: +335 612852 44. E-mail: marie.tremblay-franco@toulouse.inra.fr

Cécile Chevrier, INSERM UMR 1085 (IRSET), Université Rennes 1 - campus de Beaulieu 
Avenue du général Leclerc, 35042 Rennes Cedex, France. Tel : +332 232361 26. Fax: +332 232359 29. E-mail:cecile.chevrier@univ-rennes1.fr

Cécile Canlet, INRA - UMR 1331 TOXALIM, Bâtiment A, 180 chemin de tournefeuille, BP 93173, 31027 Toulouse cedex 3, France. Tel : +335612857 13. Fax: +335 612852 44. Email:cecile.canlet@toulouse.inra.fr

Laurent Debrauwer, INRA - UMR 1331 TOXALIM, Bâtiment A, 180 chemin de tournefeuille, BP 93173, 31027 Toulouse cedex 3, France. Tel : +335 612857 14. Fax: +335 472537 12. E-mail:laurent.debrauwer@toulouse.inra.fr

Jean-Pierre Cravedi, INRA - UMR 1331 TOXALIM, Bâtiment A, 180 chemin de tournefeuille, BP 93173, 31027 Toulouse cedex 3, France. Tel : +335 612850 02. Fax: +33561285244.E-mail:jcravedi@toulouse.inra.fr Sylvaine Cordier, INSERM UMR 1085 (IRSET), Université Rennes 1 - campus de Beaulieu Avenue du général Leclerc, 35042 Rennes Cedex, France. Tel : +332 232359 29. Fax: +332 232359 29. E-mail: sylvaine.cordier@univ-rennes1.fr

\section{Short running title:}

Metabolomics and environmental health

\section{Key-words}

Metabolomics, Environmental health, Biomarkers, System biology.

\section{Acknowledgments including grant information}

We acknowledge financial support from the EHESP School of Public health, France. 


\section{Abbreviations:}

ADI: Acceptable daily intake

BBP: Butylbenzylphthalate

DEHP: Di(ethylhexyl)phthalate

DBP: Di-n-butylphthalate

DNA: Desoxyribonucleic acid

GABA: Gamma-aminobutyric acid

GC: Gas chromatography

HILIC: Hydrophilic (ion) interaction liquid chromatography

HPLC: High performance liquid chromatography

HRMS: High resolution mass spectrometry

LDA: Linear discriminant analysis

LDL: Low density lipoprotein

MS: Mass spectrometry

NMR: Nuclear magnetic resonance

NOAEL: No observed adverse effect level

OPLS: Orthogonal projection to latent structure

PAHs: Polycyclic aromatic hydrocarbons

PCA: Principal component analysis

PLS: Partial least squares

PLS-DA: Partial least squares discriminant analysis

SVM: Support vector machines

TCA: Tricarboxylic acid

UPLC: Ultra performance liquid chromatography

VLDL: Very low density lipoprotein 


\begin{abstract}
Human beings may be exposed via their environment to multiple chemicals as a consequence of human activities and the use of man made products. Little knowledge is routinely generated on the hazards of these chemical mixtures. The metabolomic approach is widely used to identify metabolic pathways modified by diseases, drugs, or toxic exposures. This review, based on the state-of-the-art of the current applications of metabolomics in environmental health, attempts to determine whethermetabolomics can constitute an original approach to the study of associations between multiple, low-dose environmental exposure and subtoxic effects in human beings.Studying the biochemical consequences of complex environmental exposures is a challenge demanding the development of careful experimental and epidemiological designs, in order to take into account possible confounders associated with the high level of inter-individual variability induced by different lifestyles. The choice of populations studied, sampling and storage procedures, statistical tools used and system biology are worthy of consideration. Suggestions for improved experimental and epidemiological designs are discussed. This short review shows that metabolomics may be a powerful tool in environmental health in the identification of both complex exposure biomarkers directly in human populations and modified metabolic pathways, in a bid to improve understanding the environmental causes of diseases. Nevertheless, the validity of biomarkers and the relevancy of animal-to-human extrapolation remain key challenges that need to be properly explored.
\end{abstract}




\section{Introduction}

Regulations on chemicals entering the European Union market, for both domestic and professional uses, are based on risk assessment and management,on a chemical-by-chemical basis (i.e. Regulations EC 1107/2009 on pesticides or EC 1907/2006 on chemicals). Although these methods allow a comprehensive risk assessment for single chemicals for regulatory purposes, they do not permit a global risk analysis in case of different uses and multiple exposures. Such multiple exposures are characterized by various substancesand sources or routes of exposure (diet, air, oral or respiratory for example). Regulatory toxicological toolsare not suitable to the study of effects induced by the complex and low-dose mixtures that arerepresentative of human exposures, and are of limited relevance for environmental health.In this context, the development of new strategies - especially the identification of biomarkersof early effect using high-throughput approachessuch as metabolomics - is of particular importance. This bioanalytical approach entails studying the nature and quantity of potentially all metabolites produced by an organism,i.e. the detectable endogenous molecules involved in the growth and homeostasis of the organism. Themetabolic profile constitutes the ultimate step in the cellular response, and is considered the key link between genesand phenotypes(Fiehn 2002).It has come to be widely used in recent years to identify metabolic pathways modified by disease, drugs or toxic exposure, as reviewed by several authors(Lindon et al. 2004; Robertson et al. 2011). Because it has the ability to provide helpful information in the definition of markers of toxicity - as reported for example for hepatotoxicity or nephrotoxicity (Beger et al. 2010) - this approach may constitute an integrative tool to increase our understanding of the subtle metabolic disruptions induced by complex and low-dose exposures to pollutants as described in figure 1. 
Furthermore, the metabolic changes induced by these exposures may be observed directly from biological fluidsthat are easy to collect - such as urine or blood - making possible their identification directly in humans. However, these changesremain difficult to highlight,due to the high number of potentially confoundingfactors affecting the metabolome, in addition to environmental exposure (lifestyle, diet or therapeutic drug treatments for example). This is why the metabolomic approach in humans has not yet been extensively explored in the field of environmental health. In this context, this short review attempts to determine whether metabolomics can constitute an original approach to study of the associations between multiple, low-dose exposures to environmental pollutants and effects in human beings.Following a short description of the methods used in metabolomics, current applications in environmental health - in both toxicology and epidemiology - will be describedto identify the most important criteria that would be taken into account.Finally, this article addresses ways to identify key methodological challenges. This article is not intended to be a comprehensive review of the methods used in metabolomics (already available elsewhere), nor to indicate how toxicological studies may provide a corroborative evidence to support the results observed in epidemiological studies.

\section{Methods used in metabolomics}

Metabolomics combines the use of analytical and statistical toolstoidentify the metabolites that are significantly modified between groupsas shown in the flowchart presented in figure 2. Two types of metabolomicapproaches can be distinguished: untargeted metabolomics, which corresponds to a comprehensive analysis of all measurable molecules in a sample (including unknown metabolites), and targetedmetabolomics, in whicha set of chemically and biochemically characterized metabolites (such as lipids, amino acids, nucleotides, or steroids)are measured.Samples used for metabolomic studies could be any biological matrices 
(fluids such as urine, plasma, saliva, sperm, animal tissue extracts or human tissues like biopsies or placenta, for example). The most widely usedsamples are urine and plasma because theycorrespond tonon- (or minimally) invasive procedures and requirelimited, if any,pre-treatment steps, prior toanalysis.

\section{$\underline{\text { Analytical Tools }}$}

The analytical tools used in metabolomics are based on spectroscopic techniques. Currently, due to their performance for the analysis of small organic molecules and their complementary information, nuclear magnetic resonance (NMR) and mass spectrometry(MS) are the most widely used analytical techniques in metabolomics. MSis mostly used in hyphenation with different chromatography techniques (gas or high/ultra-performanceliquid chromatography, respectively GC and H/UPLC) or capillary electrophoresisfor sample separation prior to MS analysis. Thesemethods have been in use formore than 40 years e.g. forthe characterization of cellular energy metabolism or forbiofluids analyses.Sample preparation steps typically used for NMR and MS analyses have recentlybeen reviewed (Alvarez-Sanchez et al. 2010a). The main advantagesof NMR rely on the ease of sample preparation, non-destructive analysis of the sample (which may be recovered for further analyses), adaptability of the method to highthroughput analyses and the ability to provide a quantitative picture of the metabolic fingerprint. This technique wastherefore generally preferred for large-scale applications, where thousands of molecules were being measured within a short period of time. NMR allows for measurement of metabolites, typically at the $\mu \mathrm{g} / \mathrm{mL}$ level, and is less sensitive than MSwhich can detect compounds at the $\mathrm{pg} / \mathrm{mL}$ level. Besides sensitivity, the broaddiversity of ionization methods and mass analyzers(Bedair and Sumner 2008), available for MS allows identification of a large number of metabolites. The development of high-resolution MS techniques (particularly during the last ten years) has expanded the capacity for compound identification, thanks in particular to accurate mass measurements offering access to the 
elemental composition of metabolites (and their potential fragments). This feature provides invaluable information for the identification of "known unknowns" using chemical database queries. However, unlike NMR, MS sensitivity is compound-dependent and may be affected by matrix effects. Moreover,absolute quantification by MS requires specific approaches based on targeted compounds, and global profiling is often limited to semi-quantitative approaches. To a lesser extent,optical spectroscopy (e.g. near-infrared or Raman) is also used because of the simplicity of sample preparation. Each toolcan provide complementary information regarding the wide diversity of metabolite chemical structures.Comprehensive reviews on these topics are available elsewhere(Bothwell and Griffin 2011;Dettmer et al. 2007;Dunn et al. 2005;Lindon and Nicholson 2008;Ross et al. 2007) and a comparison of NMR and MS techniques was recently covered as part of a toxicometabolomics review (Bouhifd et al. 2013).

\section{Data pre-processing}

The objective of the data pre-processing stage is the achievement of extracted data corresponding to quantified spectral peaks, by converting raw data into files easily useable for statistical analyses. It includes different steps depending on the chosen analytical tool and has already been described well in the review by Madsen et al. (Madsen et al. 2010). Alignment, baseline correction, peak picking ordeconvolutionare several methods practicable for both MS and NMR techniques, and previously described (Koek et al. 2011; Theodoridis et al. 2012).In MS, resulting datasets include retention time, $\mathrm{m} / \mathrm{z}$ ratio and intensity of each detected signal. Peak identification is therefore required.Normalization can also be used to minimize bias and experimental variability induced in the analytical step. In NMR spectroscopy,it is also possible to includeabucketing step in the case of untargeted metabolomics, where spectra are reduced into consecutive spectral bins(see Ross et al. 2007 for review).Subsequent steps include scaling - when maximum variance projection methods were applied -to give the same 
weight to variables which could have different ranges. Most often, unit variance (the data matrix is divided by the standard deviation to obtain a variance of 1), mean centering (subtraction of the mean) or Pareto scaling (divided by the square root of the standard deviation) is used (see Van den Berg et al. 2006 for more information).The pre-processing steps were recently described in a comprehensive review (Molinska et al. 2012). Finally, filtering could also be used to improve data quality. A few techniques and practical examples are described in the review from Bouhifd (Bouhifd et al. 2013).

\section{$\underline{\text { Statistical tools }}$}

Spectral analyses engender hundreds of variables (peaks or spectral regions) which are highly correlated. Dimension reduction is therefore one of the main objectives of statistical analyses and is performed by multivariate methods.A large number of different statistical tools are available - in both unsupervised and supervised methods - and has been briefly described elsewhere (Madsen et al. 2010; Putri et al. 2013). For example, we canmentionprincipal component analysis (PCA), non-hierarchical or hierarchical clustering,linear discriminant analysis (LDA), partial least squares (PLS) regressions, orthogonal projections to latent structures (OPLS), and machine learning techniques such as support vector machines (SVM). PCA and PLS are most often used to study the effects of environmental pollutants on health (see next chapter).

PCA is a non-supervised method allowings visualization of the spontaneous distribution of the observation in a two dimensional plane(Bair et al. 2006);Partial least squares (PLS) regressions are supervised methods that supply a statistical model explaining individual characteristics (e.g. exposures) according to the matrix of experimental variables (i.e. metabolites)(Wold et al. 2001). Different variants exist, such as PLS-DA(PLS-discriminant analysis), widely used for modeling observations belonging to different groups (e.g. case/control or differently exposed individuals)(Waterman et al. 2009) or orthogonal PLS 
(OPLS). In OPLS, Y-predictive variability of $\mathrm{X}$ (correlated to $\mathrm{Y}$ ) is separated from $\mathrm{Y}$ orthogonal variability (uncorrelated to Y). Predictive variability is only modeled by predictive components(Trygg and Wold 2002). In all cases, the different models built should be validated. The most common methods are the use of an independent validation set or crossvalidation. A description of these methods is made elsewhere (Broadhurst and Kell 2006; Wold et al. 2001).

\section{Metabolite identification and biological interpretation}

Metabolite identification plays a key role in the interpretation of the metabolomics research results. Different approaches are needed depending on the analytical tool chosen butspectral library searching on the Internet is a common step. For example, the Madison metabolic consortium database (MMCD, http://mmcd.nmrfam.wisc.edu/), the Human Metabolome Database (HMDB, http://www.hmdb.ca/), the Biological Magnetic Resonance Data Bank (BMRB, http://www.bmrb.wisc.edu/metabolomics/)and the Kyoto encyclopedia of genes and genomes (KEGG, http://www.genome.jp/kegg/) are widely used.Werner et al. (2008) reviewed databases from mass spectrometry data used for metabolome annotation.More recently, other authors have reviewed the huge development of the metabolome databases (Fukushima and Kusano, 2013), especially the HMDB which has been considerably expanded,and includes now more than 40,000 annotated metabolites (Whisart et al. 2009; 2013).

Unknown metabolites have to be identified using MS/MS or NMR structural characterization tools. When a chemical structure is suggested, confirmation can be obtained by comparison with the spectra of authentic standards. When potential metabolites from exogenous contaminants are suggested, it is also possible to carry out short experimentations in laboratory mammals exposed to the parent compounds, so asto unambiguously characterize their metabolites by plasma or urinary analysis (Jamin et al. 2013). 
Two dimensional NMR spectra are also required as an aid to spectral assignment and to confirm the identity of metabolites.Once candidate biomarkers have been identified, the final step is to decipher the main metabolic pathways involved in the modifications observed, and suggest biological hypotheses in accordance with the involvement of the modified metabolites in biochemical pathways (amino-acids synthesis, energetic metabolism, fatty acids oxidation, etc.).

In order to fully integrate these data, mechanistic interpretation has to be achieved at the metabolic network level. Metabolic network gathers all the metabolic reactions an organism can perform into a single mathematical model. For Humans, a compartmentalised model publicly available (Duarte et al. 2007) contains 3311 reactions and 2766 metabolites. The aim is to computationally decipher, among all these reactions and metabolites, the ones involved in the metabolic response of the organism when exposed to contaminants. To do so, algorithms have been developed which extract relevant sub-networks based on network topology and biomarkers (Jourdan et al. 2010).

\section{Current applications in environmental health}

Animal experiments and epidemiological studies are commonly used to assess potential factors that can affect human health, and in particular the effect of chemical exposure as a risk factor. In this context, comparing various exposure groups in animals or humanscan determine potential links between an environmental factor and a health effect. In the field of toxicology, metabolomics is used to identify candidate biomarkers of exposure and effects. Many toxicological studies demonstrate that metabolomics is a powerful method for detecting changes in the metabolome of exposed individuals. The identification of discriminant metabolites between groups is made easier by the possibility of controlled conditions: interindividual variability among tested animals is reduced by the use of similar species, strains, 
individuals and laboratory conditions.Most of the toxicological studies have been published within the context of drug development(Beger et al. 2010;Robertson 2005). In recent years, some authors have focused on environmental pollutants, in particular endocrine disruptors such as phthalates (plasticizers widely found in our environment) and pesticides. Table 1 describes the main studies carried out on animal models, the methodological approaches used, and the main results. Sumner et al. (2009) showed correlations between developmental outcomes in rat pups exposed to benzylbutylphthalate (BBP) during pregnancy and modification of the metabolic pathways in dams and pups. Modifications included metabolites derived from citrate cycle and amino acid catabolism. Changes were observed in urine by NMR spectroscopy and subsequent PCA and PLS-DA analyses. It has been suggested that di(ethylhexyl)phthalate (DEHP) exposure could modify several aminoacid concentrations (glutamine, valine, tyrosine/phenylalanine, isoleucine) in the plasma and liver extracts frommale mice (NMR analyses, OPLS statistical data treatment), leading the authors to suggest a new hepatic pathway targeted by DEHP exposure in mice explaining its potential for endocrine disruption (Eveillard et al. 2009). In utero,DEHP was also shown to affect lipid profiling in the fetal rat brain (Xu et al., 2007), which may lead to aberrant neurodevelopment. More recently, Xia and co-workers (2011) combined the analyses of multiple biological matrices, including maternal serum, placenta and fetal brain tissue in order to evaluate the teratogenic effects of di- $n$-butylphthalate (DBP) in mice. By means ofGC time-of-flight MS combined with PLS-DA, these authors have shown that DBP disrupted maternal and fetal metabolic profiles, altering citrate cycle, amino acid, purine and lipid metabolism.Van Ravenzwaay et al. (2010) used metabolomics based on MS analyses of plasma samples to investigate possible interactions resulting from combined exposure to DEHP and DBP in rats. They showed that simultaneous exposure to high dosages of the mixture ( $3 \mathrm{mg} / \mathrm{kg}$ feed) over a period of 28 days resulted in a profile that was significantly different from the individual 
compounds. However, these effects were less than additive and inconsistent changes were observed at lower doses. Co-exposure to DEHP and aroclor 1254 was found to disturb lipid metabolism, leading to an accumulation of lipid in the liver(Zhang et al. 2012). Tryptophan and phenylalanine metabolisms were also disturbed in this case. Recently, Cabaton et al. (2013) hypothesized that the metabolism of mice perinatally exposed to bisphenol A may be disrupted. After subcutaneous low-dose exposure (0.025 to $25 \mu \mathrm{g} / \mathrm{kg} \mathrm{BW} /$ day) to pregnant mice from gestational day 8 through day 16 of lactation, they showed a disruption in the glycolysis, glycogenesis and energy metabolism both at postnatal day 2 and 21.They also confirmed brain development alterations suggested in the literature for bisphenol A(decrease in glutamate and GABA concentrations in brain samples at postnatal day 21) (Cabaton et al. 2013).

Some publications draw attention to the potential of metabolomics in pesticide research, including toxicological issues (see Aliferis and Chrysayi-Tokousbalides 2011 for review). They show promising results in terms of (eco)-toxicological risk assessment of bioactive compounds using model organisms representative of different levels of organization. Kim and co-workers investigated the metabolic profile of methoxychlor, an organochlorine insecticide known to be an endocrine disrupter. The NMR analyses of urine samples from female rats showed a decrease of acetate, benzoate, lactate, glycine and alanine in a dose-dependent manner(Kim et al. 2009).A recent study showed the impact of endosulfan, another organochlorine insecticide, on the metabolic profile of blood, bone marrow and liver in mice orally exposed for 11 weeks to a level corresponding to the acceptable daily intake (ADI). A decrease in plasma LDL, VLDL and choline concentrations and an increase in plasma glucose levels were observed, associated with a potential oxidative disturbance in the liver (Canlet et al. 2013). 
The same team also showed modification of amino acid metabolism, citrate cycle, urea cycle and glucose metabolism in mice orally exposedto the same dose of endosulfan combined with atrazine and chlorpyrifos (exposure levels corresponding to their respective ADI).Wang and co-workers $(2009,2011)$ investigated the metabolic profiles of rats following exposure to chlorpyrifos and carbaryl, two anticholinesterase insecticides known to be neurotoxic in mammals. Serum analyses suggest that their exposure(alone or in combination) could cause a disturbance in energy and fatty acid metabolism in the liver mitochondria (Wang et al. 2009). Metabolomic analysis of urine confirmed these conclusions, especially in terms of impact on the energy metabolism (Wang et al. 2011). The same group recently investigated the metabolomic responses of rat to propoxur, another anticholinesterase insecticide (Liang et al. 2012a; 2012b). The NMR analysis of urine samples suggeststhe induction of oxidative stress, and alteration inthe energy metabolism and lipid metabolism (enhancement of ketogenesis and fatty acid beta-oxidation) in the liver contributing to the hepatotoxicity of this insecticide, as previously shown for chlorpyrifos and carbaryl (Wang et al. 2009; 2011). They also studied the effect of combined exposures to oganophosphorus insecticides (dichlorvos or propoxur) with pyrethrinoïds (deltamethrin or permethrin), and showed that, even at doses not exhibiting any clinical or physiopathological effects, organophosphorus and pyrethrinoids, tested individually or in combination, were able to disrupt the energy metabolism of the liver andinduce potential nephrotoxicity (Wang et al. 2013; Liang et al. 2012c). Similar findings were published after oral and chronic exposures (drinking water) to dichlorvos in rats (disturbances in the carbohydrate and fatty acid metabolism, anti-oxidant system) (Yang et al. 2011; 2013). Other authors investigated the metabolic changes in rats induced by chronic oral exposure (viadrinking water) to dimethoate, an organophosphorus insecticide. They found significant changes in the urinary and plasmatic metabolic UPLC-MS fingerprints, especially for L-tyrosine, citric, uric and suberic acids, glycylproline, allantoin, isovalerylglutamic acid 
and lipids (as well as urinary organophosphorous metabolites). The authors suggested a link between these changes and the impairment of the hepatic and nervous systems (Feng et al. 2012). A series of other chronic studies in rats on organophosphorus insecticides also showed renal and perturbed glucose, nucleic acid, and protein metabolism (for acephate) (Hao et al. 2012), disturbance in energy and lipid metabalolism, oxidative stress, and DNA damage (for phorate) (X. Sun et al. 2012).This series of studies demonstratesthe ability of the metabolomics approach to identify metabolic pathways modified by environmental contaminants and suggest hypotheses about their potential mechanism of action. Moreover, as was observed for BBP, DBPand some organophosphorus pesticides, metabolomics could, in some cases, be more sensitive on a dose scale than histopathology orclinical blood biochemistry in recognizing early toxicological events.

Recently, several other contaminants were investigated in rodents. Neerathilingam et al. (2010) showed a disturbance of the energy metabolism with changes in citrate cycle metabolites in 24-hour urine collection of rats exposed to tributylphosphate (an organophosphorous widely used as a solvent, extractant or plasticizer). The adverse renal response to perfluorododecanoic acid, a perfluorinated carboxylic chemical, was also identified in male rats as contributing to disorders in glucose and amino acid metabolism $(\mathrm{H}$. Zhang et al. 2011).A modification of the urinary profile of mice subchronically exposed to benzene was described, even at a dose which does not elicit changes in body weight or blood parameters (R. Sun et al. 2012). Changes related to glutathione, TCA cycle and amino acids metabolism were observed in the urinary profile of rats exposed to acrylamide (J. Sun et al. 2010).

These publications indicate that metabolomics represents a promising tool for studying contaminant toxicity and mode of action, as well as for investigating the effects of mixtures of 
xenobiotics. Nevertheless, in the case of environmental applications, most toxicological studies suffer from a lack of similarities with human exposure conditions, in particular through the use of high doses of a single chemical or simple mixture.Few low-dose studies are available in the case of chemical mixtures. For instance, Du et al. investigated the effect of a mixture of low-doses (corresponding to the NOAEL in a two-year study) of 4 organophosphorus pesticides (dichlorvos, dimethoate, acephate and phorate). They showed disturbance in energy and lipid metabolism, oxidative stress, and DNA damage (Du et al. 2013). Mehri et al. investigated the effect in mice of a mixture of low-doses (derived from their respective acceptable daily intakes) of six pesticides frequently found in fruits and vegetables grown in France and showed a metabolic signature linked to oxidative stress and glucose regulation (Merhi et al. 2010). Likewise, the metabolic dysfunction of contaminated lake water, which is an important drinking water source for the nearby city (in China)was investigated inmale mice. The water contamination includes polycyclic aromatic hydrocarbons (PAHs) and organochlorine insecticides. The study found that its ingestion induced liver damage with alterations in energy and amino acid metabolism(Y. Zhang et al. 2011).

Besides this metabolic profiling obtained in urine, plasma or tissue extracts from mammals exposed either orally or subcutaneously, more mechanistic studies are implemented on cell lines, both in animals (for example, neuronal cultures exposed to methylmercury and mercury chloride) (van Vliet et al. 2008) and in humans (for example, lung epithelial cells exposed to cigarette smoke or hepatocellular carcinoma-derived cells exposed to ammonia) (Vulimiri et al. 2009; Shintu et al. 2012).

Given the limitations of experimental toxicology, the best strategy for the identification of metabolic disturbance in environmental health will be to set up studies directly targeted on the human populations. Most metabolomics studies carried out in humans focus on clinical 
research and the identification of biomarkers for early detection in pathologies such as cancer, heart disease or adverse pregnancy outcomes(Diaz et al. 2011;Kenny et al. 2008;Mamas et al. 2011; Kim et al. 2010). These studies are based on the comparison of a small number of people affected by the disease againsthealthy individuals. They are very promising in terms of the discovery of biomarkers to aid disease diagnosis, because predetermined pathologies have a major influence on the metabolic pathway of an organism, facilitating identification of discriminant metabolites between cases and controls. Environmental epidemiology aims at finding subtle metabolic disturbances resulting from chronic low-level exposure to multiple pollutants. Metabolomics is not very developed in this field of research because metabolic modifications expected with environmental exposures are thought to be minor in comparisonwith the inter-individual variability that can be observed in a human population. To our knowledge, only four studies have taken an interest in modification in urinary or blood metabolic profiles associated with human environmental exposures. The first of these concerned 51 workers exposed to welding fumes in Taiwan. The study has shown an increase of metabolites involved in inflammatory and oxidative tissue injury processes, especially glycine, taurine and betaine (Kuo et al. 2012). The second was interested in urinary metabolic profiles in 178 human volunteers living near a source of environmental cadmium pollution in Great Britain. Several metabolites involved in mitochondrial metabolism (citrate, 3hydroxyisovalerate and 4-deoxy-erythronic acid) and amino acid metabolism (dimethylgycine, creatine and creatinine) were associated with cadmium exposure, suggesting oxidative stress that was confirmed by an increase ofurinary 8-oxo-deoxyguanosine(Ellis et al. 2012). The third focused on 83 pregnant women living in a French agricultural area (Brittany). As a surrogate of environmental pesticide exposure, the surface area of land dedicated to agricultural cereal activities in their towns of residence was used to categorize the women into three groups. The study showed modifications to the urinary metabolites 
involved in oxidative stress and energy metabolism (an increase in glycine, threonine, lactate and glycerophosphocholine and a decrease in citrate) in the most exposed women after adjusting for the main potential confounders (age, body mass index, parity and smoking) (Bonvallot et al. 2013). The largestof these studies was a large-scale epidemiological study (includingmore than 4,000 individuals), which was conducted to investigate metabolic variations within four different population groups with different dietary patterns (Asian, North-American, and European). The urinary metabolic excretion patterns were discriminated between populations having contrasting lifestyles, diets and pathology risk factors, in particular blood pressure and cardiovascular disease (Holmes et al. 2008). Such a large-scale metabolic approach could be very useful in identifying the main interactions between lifestyle, diet, genetics and environmental exposures.

\section{Discussion}

Metabolomic studies in environmental healthmay represent a good way of highlighting new biomarkers linked with pollutant exposures and suggesting hypotheses for a better understanding ofthe mechanistic pathways associated with metabolic changes. This short review allows identification of some issues in order to come up with an ideal design from an environmental health perspective: this ideal design should be as similar as possible to an experimental situation where the only modifying factor would be environmental exposure, i.e. without confounding factors. Both toxicology and epidemiology have advantages and limitations. In toxicology, the controlled conditions of very simple mixtures limit variability and confusion factors - but the difficulty lies in getting closer to the real human exposures (number of pollutants and levels of exposure). Epidemiology allows the complexity of human exposures to be taken into account yet requires the definition of strategies aimed at reducing the number of confoundingfactors. These observations involvethe development of new 
original environmental health strategies that combine both human and animalstudies. The main challenges in study design, recruitment of individuals or definition of exposure groups, sampling strategy, statistical tools used, pathway analysis and system biology are briefly described below.

Study design, recruitment of individuals and definition of exposure groups

The use of metabolomics in environmental epidemiology requires an improvementinexperimental protocols to optimizereliability, reproducibility, and sensitivity. One solutionis the use of large-scale studies such as metabolome-wide association studiesor metabolic phenotyping for which optimal protocols (sampling strategies, analyses and processing) have been largely described elsewhere (Bictash et al. 2010;Dumas et al. 2006).The interest of these studies mainly lies in the possibility of studying both phenotypic and cultural/environmental factors (especially diet). At this scale, it is possible to link metabolic profiling with the presence of risk factors or disease. For example, in the study from Holmes et al. (Holmes et al. 2008), cultural differences and diet could be considered as a surrogate for environmental differences, without acknowledgment of direct contaminant exposures. The same issues could be expressed for environmental epidemiology on a smaller scale. Furthermore, in this case, three main challenges may be described:

Limitation of the inter and intra-individual variability within groups being compared: the population studied should be sufficiently homogeneous to reduce uncontrolled variability, especially in terms of diet. This could be achieved through the choice of culturally-similar individuals (Lenz et al. 2004) having the same gender, age and physiological conditions such asbody mass index, for example - and without specific pathologies or medication.

Limitation of co-exposure and other confounding factors: this could be achieved by making all co-variables - such as diet (the most important co-variable in environmental health) homogeneous, for example by choosing culturally-similar individuals. In addition, it is 
advisedthat the number of individuals be high enough to take into account non-controllable variability. But giving a minimal number of subjects is almost impossible in metabolomics because of poor knowledge of variability of endogenous metabolites in all biological fluids, cells or tissues.

The choice of a contrasted environmental exposure between groups of individuals: in this case, in environmental epidemiology, geographical indicators may be used as a surrogate for complex and multiple exposures, since some studies have shown that residential proximity to anthropic activities could increase exposure to pollutants. This is well established with pesticides exposure and agricultural area, as shown by several authors (Jamin et al. 2013; Munoz-Quezada et al. 2012; Gunier et al. 2011; Bradman et al. 2011). But the measurement of environmental exposures remains a challenge. Exposure should be assessed in different ways, associating, for example, biological measurements with geographical indicators.In toxicology, the challenge remains in the definition of exposures as similar as possible to human exposures, taking into account their complexity in terms of both number of pollutants and choice of dose levels. Few toxicological studies are designed using these hypotheses (Cabaton et al. 2013; Zhang Y. et al. 2011; Mehri et al. 2010).

\section{Biological sampling strategy}

Type of biological fluid and sampling and storage conditions are essential to the design of sampling strategy(Alvarez-Sanchez et al. 2010b). Blood and urine metabolic profiles are now preferred over other biological fluids because they embody a large number of metabolic pathways representative of the whole metabolism of an organism. Theyare better studied. Other biological fluids such as saliva or hair are becoming emerging media, due to the noninvasiveness of their sampling procedures (Zhang et al. 2012; Bessonneau et al. 2013; Neyraud et al. 2013). The moment of collection for each individual should be as similar as possible since there is considerable intra-individual variability depending on time of day or 
night, especially for urine. Finally, storage conditions must be closely controlled to avoid microbial contamination or degradation, and should be the same as for all samples analyzed in orderto reduce inter-individual variability.Few studies have directly discussed storage conditions for metabolomics use. Most of these showed low metabolic degradation where samples are frozen immediately, or at most 24 hours followingcollection at ambient temperature (Maher et al. 2007). This was confirmed by Peakman and Elliott (2008) who tested sample stability at $4^{\circ} \mathrm{C}$ and $18^{\circ} \mathrm{C}$ in different studies, and showed integrity of the samples maintained at $4^{\circ} \mathrm{C}$ at least up to 24 hours (Peakman and Elliott, 2008). Dunn et al. (2008) also showed that analytical variance was of the same magnitude as variance observed between samples stored at $4^{\circ} \mathrm{C}$ for 0 or 24 hours (no statistical significant changes between the 2 storage conditions) (Dunn et al. 2008). Likewise, a long period of freezing $\left(-40^{\circ} \mathrm{C}\right.$ for urine and $-80^{\circ} \mathrm{C}$ for blood, for 9 months) does not appearto have any impact on the metabolic profile (Beckonert, 2007). Recently, Hebels et al. studied the influence of long-term storage of blood samples for omics analysis in environmental health research. No trend was observed in relation to the storage temperature after immediate thawing (storage at $-80^{\circ} \mathrm{C}$ from several weeks to several months) (Hebels et al. 2013).Although one study showed minimal effects of storage temperature (overa period of one week) on human plasma lipids profiles (tested conditions: $4^{\circ} \mathrm{C},-20^{\circ} \mathrm{C}$ and $-80^{\circ} \mathrm{C}$ ) (Zivkovic et al. 2009), Deprez et al. showed more changes following storage at $4{ }^{\circ} \mathrm{C}$ or at room temperature on rat plasma samples. The changes observed could be due to lipid hydrolysis (Deprez et al. 2002). However, even though a few studies showed significant modifications of metabolic profiles in plasma, in order to define the most rigorous study design, it would be preferable to have immediate freezing of biological samples, directly after collection, to prevent changes and decrease possible variability observed between samples.

\section{Analytical methods}


In terms of their advantages and limitations, both NMR and MS techniques appear to be highly complementary.On the one side, the ease of sample preparation and rapid analysis time provided by NMR techniques is well adapted to high throughput fingerprinting, and on the other, the high sensitivity of MS techniques and their ability to measure more metabolites allows for extended metabolome coverage. Indeed, NMR allows for the measurement of major metabolites (i.e. metabolites in the field of micromolar concentrations) such as amino acids, organic acids, carbohydrates and lipids. MS is able to evidence minor metabolites such as steroids, hormones, neurotransmitters or other trace compounds, which may be present at concentrations as low as picomolar. On the other hand, NMR provides access to highly polar metabolites (sugars, sugar phosphates, etc) which are often difficult to handle using the universal reversed phase C18-based LC-MS methods, and which may require specific analytical strategies such as hydrophilic (ion) interaction liquid chromatography (HILIC) MS or capillary electrophoresis - MS coupling (Idborg et al. 2005; Cubbon et al. 2010; Ramautar et al. 2013). It therefore appear relevant, in order to identify dynamic pathways of toxicity, to associate the two technologies in metabolomics for environmental health. As discussed by Bouhifd et al., the choice of an analytical tool is generally a compromise between sensitivity and selectivity (Bouhifd et al. 2013). However, the combined use of NMR and MS could enable characterization of many more metabolites - not only those related to the functioning of the organism (endogenous metabolites), but also those generated by environmental exposure (traces of pollutants). Indeed, in addition to the identification of endogenous metabolites, metabolomics is already used in the detection and identification of metabolites coming from xenobiotics, using targeted or semi-targeted techniques. For example, conjugated metabolites of bisphenol A were unambiguously characterizedin plasma, urine and testis from laboratory rodents orally exposed (Lacroix et al. 2011; Chen et al. 2012) using targeted profiling.Semi-targeted profiling was used to characterize pesticide metabolites 
in the urine of pregnant women living in a French agricultural area. Based on a list of numerous pesticides commonly used on French crops, and using UHPLC coupled to HRMS, several metabolites from two fongicides (azoxystrobin and fenpropimorph) were identified in the urine of thosewomen livingin areas where cereal cropspredominate(Jamin et al. 2013). This approach can be considered to bea first step in the characterization of an "exposome", since it was described for the first time by Wild (2005) and defined as the totality of all exposure over a lifetime. The term "all exposure" refers to a global approach taking into account not only a variety of sources such as hazardous agents (chemical, physical, biological) as well as social characteristics considered "external exposures" - but also all measurable biomarkers (metabolites, adducts, biotransformation products, etc.) considered as markers of "internal exposures". Rappaport defined two generic approaches for characterizing the exposome: the bottom-up approach characterizing external exposure (environmental contamination and exposure assessment), which is useful in improving prevention strategies in public health, and the top-down approach, which uses untargeted omics techniquesto identify biomarkers in biological fluids, and is useful inimproving knowledge about the causes of human disease (Lioy and Rappaport, 2011; Rappaport, 2011).In characterizing the global exposure of human populations at different stages of life, metabolomics could make the connection between "external" and "internal" exposures at critical periods of life, as it was described by T.J. Athersuch (Athersuch, 2012). This author and his collaborators proposed several keys to the development and application of omics technologies (including metabolomics) in environmental epidemiology (Vineis et al. 2013).

\section{Statistical analysis}

In environmental epidemiology, supervised methods could be preferred due to the high level of variability within a human population, often inducing a lack of separation with unsupervised methods such as PCA (Miller, 2007). For example, cluster analysis and PLS 
have been already shown to be successful in human studies (Ellis et al. 2012;Bonvallot et al. 2013; Holmes et al. 2008). However,application of a preliminary PCA would be usefulinhighlighting the spontaneous separation of outliers.

\section{Biomarker identification}

Currently, metabolite identification remains a key challenge in metabolomics. Even though (as previously mentioned) numerous databases have been established and developed in recent years, none of these offers overall coverage of the metabolome. Searching against all databases is necessary, but comparison of information from one database to another is difficult, due to the varying data format or data recovery methods used. To improve the biomarker identification step, many bio-informatics toolshave been developed, covering both MS (Zhou et al. 2012; Li et al. 2013) and NMR spectra (Tulpan et al. 2011).

\section{Biological interpretation}

Acquisition of information - on the quantities of the different metabolites found in biological fluids in normal conditions, and factors influencing their levels in biological fluids in order to be able to detect subtle disturbances related to environmental factors - is an important challenge(Vlaanderen et al. 2010). Many studies have been published concerning the impact of certain physiological parameters in the metabolic profiles of a normal healthy population. The main factors include, but are not limited to: age (D'Adamo et al. 2010;Gu et al. 2009), gender (Bertram et al. 2009; Kochhar et al. 2006), body mass index (Bertram et al. 2009) and also diet ingested 24 hours prior to collection(Lenz et al. 2004). These studies are very helpful in improving knowledge about metabolic variability between healthy individuals, and could be very useful in environmental epidemiology. Additional mechanistic information onthe metabolic pathways involved in the toxicity of complex mixtures should be providedfromanimal experiments. Indeed, the use of environmental health metabolomics in humans is still exploratory and it requires the creation of a connection between metabolic 
modifications which may be observed, early toxicity and adverse effects on the organism, as made by Ellis et al. with the association between modified urinary metabolic profiles and proteinuria (Ellis et al. 2012). These connections are easier to determine in animals with the use of different doses scales, allowing to identify adverse effects, early toxic effects, and metabolic changes in a same experiment. In this case, given the great diversity of environmental exposures, the challengeslie in the choice of exposure groups and model chemical mixtures that are as close as possible to human exposure andin finding out how the various metabolites are connected together in order to regulate cell functioning. The question that needs to be addressed is: what is the biological and health significance in the whole organism of the observed metabolic changes?

The understanding of biological networks is of great interest but still needs significant development, particularlyin computational tools (Kholodenko et al. 2012). Progress has been made recently in the reconstruction of the metabolic networks of biological systems, and the mechanisms triggering the shifts of these metabolic networks following exposure to model contaminants. For this, NMR was used, but also high-resolution MS, which grants access to a larger set of metabolites, and makes it possible to characterize their exact mass. In order to study the modulation of metabolic networks and to gain a better understanding of the mechanisms of action of low doses of contaminants, computational models of these networks have been developed (Jourdan et al., 2010). In parallel, there has been considerable progress in the development of bioinformatic tools and databases (e.g. KEGG (Kanehisa and Goto, 2000) and HMDB (Wishart et al. 2007, 2009, 2013)) making metabolomics studies much more quantitative and far more extensive in terms of metabolic coverage. Recent biochemical databases provide information about the interconnectivity of metabolism which can be automatically polled using metabolomics secondary analysis tools. Starting with lists of altered metabolites, there are two main types of analysis: enrichment analysis computes which 
metabolic pathways have been significantly altered whereas metabolite mapping contextualizes the abundances and significances of measured metabolites into network visualizations (Booth et al. 2013).

In this case, targeted metabolomics on particular metabolic pathways could be an interesting perspective in order to explain more in-depth environmental contaminant mechanisms of action.

Finally, this short review has shown that although numerous studies are published in the field of toxicology to study the mechanisms of action of environmental pollutants, little epidemiological data gives rise to a comprehensive knowledge of what may be observed in humans. The issue of animal-to-human extrapolation - well-studied in hazard characterization- is still topical, and remains a challenge in metabolomics. A new research strategy is needed in order to understand the significance of the modifications observed in a metabolic profile as well as the validity of the biomarkers identified in toxicology. The role of in vitro metabolomics may have the capacity to meet this challenge with the development of human cell assays and the comparison of the mechanisms of action of environmental pollutants between species.

In conclusion, metabolomics may be a powerful tool in environmental health for two main reasons: 1) the identification of complex exposure biomarkers, directly in human populations, or the characterization of "internal exposures" as an approximation of the exposome; and 2) the identification of modified metabolic pathways allowing the suggestion of hypotheses on mechanisms of toxicity and in order to better understand the environmental causes of diseases. Although it is not yet well used in humans, there is potential for the development of this technique, associated with classical environmental epidemiology and toxicology, as was shown by the first studies published in this field in recent years.Numerous key challenges 
were highlighted that needed further research - including recruitment of individuals and definition of exposure groups, sampling strategies, analytical and statistical techniques, metabolite identification and biological/ toxicological interpretation. In addition, the relevance of animal-to-human extrapolation remains a dominant issue.

\section{References}

Aliferis K.A. and M. Chrysayi-Tokousbalides. 2011. Metabolomics in pesticide research and development: review and future perspectives. Metabolomics 7(4):35-53.

Alvarez-Sanchez B., F. Priego-Capote, and M. Luque de Castro. 2010a. Metabolomics analysis II. Preparation of biological samples prior to detection. TrAC Trends Anal Chem 29: $120-127$.

Alvarez-Sanchez B., F. Priego-Capote, and M. Luque de Castro. 2010b. Metabolomics analysis I. Selection of biological samples and practical aspects preceding sample preparation. TrAC Trends Anal Chem 29: 111-119.

Athersuch T.J. 2012. The role of metabolomics in characterizing the human exposome. Bioanalysis 4(18):2207-2212.

Bair E., T. Hastie, D. Paul, and R. Tibshirani. 2006. Prediction by Supervised Principal Components. JASA 101: 119-137.

Bedair M. and L.W. Sumner. 2008. Current and emerging mass-spectrometry technologies for metabolomics. TrAC Trends Anal Chem 27: 238-250.

Beger R.D., J. Sun, and L.K. Schnackenberg. 2010. Metabolomics approaches for discovering biomarkers of drug-induced hepatotoxicity and nephrotoxicity. Toxicol Appl Pharmacol 243: $154-166$

Bertram H.C., J.O. Duus, B.O. Petersen, C. Hoppe,A. Larnkjaer, L. Schack-Nielsen, C. Molgaard,and K. Michaelsen. 2009. Nuclear magnetic resonance-based metabonomics reveals 
strong sex effect on plasma metabolism in 17-year-old Scandinavians and correlation to retrospective infant plasma parameters. Metabolism 58: 1039-1045.

Bessonneau V., B. Bojko, and J. Pawliszyn. 2013. Analysis of human saliva metabolome by direct immersion solid-phase microextraction LC and benchtop orbitrap MS. Bioanal 5(7):783-792.

Bictash M., T.M. Ebbels, Q. Chan, R.L. Loo, I.K.S. Yap, I.J. Brown, M. de Iorio, M.L. Daviglus, E. Holmes, J. Stamler, J.K. Nicholson, and P. Elliott. 2010. Opening up the "Black Box": Metabolic phenotyping and metabolome-wide association studies in epidemiology. J Clin Epidemiol 63: 970-979.

Bonvallot N., M. Tremblay-Franco, C. Chevrier, C. Canlet, C. Warembourg, J-P Cravedi and S. Cordier.2013. Metabolomics Tools for Describing Complex Pesticide Exposure in Pregnant Women in Brittany (France). PLoS ONE 8(5): e64433. doi:10.1371/journal.pone.0064433.

Booth S.C., A.M. Weljie, R.J. Turner. 2013. Computational Tools for the Secondary Analysis of Metabolomics Experiments. Comp and Struct Biot Journal 4(5):e201301003.

Bothwell J.H.F. and J.L. Griffin. 2011. An introduction to biological nuclear magnetic resonance spectroscopy. Biological Reviews 86: 493-510.

Bouhifd M., T. Hartung, H.T. Hogberg, A. Kleensang, and L. Zhao. 2013. Review: Toxicometabolomics. J Appl Toxicol. doi: 10.1002/jat.2874.

Bradman A., R. Castorina, D. Boyd Barr, J. Chevrier, M.E. Harnly, E.A. Eisen, T.E. McKone, K. Harley, N. Holland and B. Eskenazi. 2011. Determinants of organophosphorus pesticide urinary metabolite levels in young children living in an agricultural community. Int $\mathrm{J}$ Environ Res Public Health 8: 1061-1083.

Broadhurst D. and D.B. Kell. 2006. Statistical strategies for avoiding false discoveries in metabolomics and related experiments. Metabolomics 2: 171-196. 
Cabaton N., C. Canlet, P.R. Wadia, M. Tremblay-Franco, R. Gautier, J. Molina, C. Sonnenschein, J-P. Cravedi, B.S. Rubin,A.M. Soto, D. Zalko. 2013. Effects of Low Doses of Bisphenol A on the Metabolome of Perinatally Exposed CD-1 Mice. Environ Health Perspect doi:10.1289/ehp.1205588.

Canlet C., M. Tremblay-Franco, R. Gautier, J. Molina, B. Métais, F. Blas-Y-Estrada, L. Gamet-Payrastre. 2013. Specific Metabolic Fingerprint of a Dietary Exposure to very Low Dose of Endosulfan. J Toxicol 545802. http://dx.doi.org/10.1155/2013/545802

Chen M., B. Xu, W. Ji, S. Qiao, N. Hu, Y. Hu, W. Wu,L. Qiu, R. Zhang, S. Wang, Z. Zhou, Y. Xia, X. Wang. 2012. Bisphenol A alters n-6 fatty acid composition and decreases antioxidant enzyme levels in rat testes: a LC-QTOF-based metabolomics study. PLoS One7(9):e44754.

Cubbon S., C. Antonio, J. Watson, J. Thomas-Oates. 2010. Metabolomic applications of HILIC-LC-MS. Mass Spectrom. Rev. 29: 671-684.

D'Adamo P., S. Ulivi, A. Beneduci, G. Pontoni, G. Capasso, C. Lanzara, G. Andrighetto, U. Hladnik, V. Nunes, M. Palacin, and P. Gasparini. 2010. Metabonomics and population studies: age-related amino acids excretion and inferring networks through the study of urine samples in two Italian isolated populations. Amino Acids 38: 65-73.

Demur C., Métais B., Canlet C., Tremblay-Franco M., Gautier R., Blas-Y-Estradas F., Sommer C., gamet-Payrastre L. 2013. Dietary exposure to a low dose of pesticides alone or as a mixture: the biological metabolic fingerprint and impact on hematopoiesis. Toxicology 308:74-87.

Deprez S., B.C. Sweatman, S.C. Connor, J.N. Haselden, and C.J. Waterfield. 2002. Optimisation of collection, storage and preparation of rat plasma for 1H NMR spectroscopic analysis in toxicology studies to determine inherent variation in biochemical profiles. J Pharm Biomedical Analysis 30: 1297-1310. 
Dettmer K., P.A. Aronov, and B.D. Hammock. 2007. Mass spectrometry-based metabolomics. Mass Spectrom Rev 26: 51-78.

Diaz S.O., J. Pinto, G. Graça, I.F. Duarte, A.S. Barros, E. Galhano, C. Pita, M. Almeida, B.J. Goodfellow, I.M. Carreira, and A.M. Gil. 2011. Metabolic biomarkers of prenatal disorders: an exploratory NMR metabonomics study of 2nd trimester maternal urine and blood plasma. $\mathrm{J}$ Proteome Res 10: 3732-3742.

Du L., H. Wang, W. Xu, Y. Zeng, Y. Hou, Y. Zhang, X. Zhao, and C. Sun. 2013. Application of Ultraperformance Liquid Chromatography/Mass Spectrometry-Based Metabonomic Techniques to Analyze the Joint Toxic Action of Long-term Low-Level Exposure to a Mixture of Organophosphate Pesticides on Rat Urine Profile. Toxicol Sciences. doi: 10.1093/toxsci/kft091.

Duarte N.C., S.A. Becker, N. Jamshidi, I. Thiele, M.L. Mo, T.D. Vo, R. Srivas, and B.O. Palsson. 2007. Global reconstruction of the human metabolic network based on genomic and bibliomic data. Proceedings of the National Academy of Sciences of the United States of America, 104(6): 1777-1782.

Dumas M.E., E.C. Maibaum, C. Teague, H. Ueshima, B. Zhou, J.C. Lindon, J.K. Nicholson, J. Stamler, P. Elliott, Q. Chan,and E. Holmes. 2006. Assessment of analytical reproducibility of 1H NMR spectroscopy based metabonomics for large-scale epidemiological research: the INTERMAP Study. Anal Chem 78: 2199-2208.

Dunn W.B., N.J.C. Bailey, and H.E. Johnson. 2005. Measuring the metabolome: current analytical technologies. Analyst 130: 606-625.

Dunn W.B., D. Broadhurst, D.I. Ellis, M. Brown, A. Halsall, S. O'Hagan, I. Spasic, A. Tseng and D.B. Kell. 2008. A GC-TOF-MS study of the stability of serum and urine metabolomes during the UK Biobank sample collection and preparation protocols. International Journal Of Epidemiology 37 Suppl 1: i23-i30. 
Ellis J., T. Athersuch, L. Thomas, F. Teichert, M. Perez-Trujillo,C. Svendsen, D. Spurgeon,R. Singh,L. Jarup,J. Bundy, and H. Keun. 2012. Metabolic profiling detects early effects of environmental and lifestyle exposure to cadmium in a human population. BMC Medicine 10: 61.

Eveillard A., F. Lasserre, M. de Tayrac, A. Polizzi, S. Claus, C. Canlet, L. Mselli-Lakhal, G. Gotardi, A. Paris, H. Guillou, P.G.P. Martin, and T. Pineau. 2009. Identification of potential mechanisms of toxicity after di-(2-ethylhexyl)-phthalate (DEHP) adult exposure in the liver using a systems biology approach. Toxicol Appl Pharmacol 236: 282-292.

Feng Z., X. Sun, J. Yang, D. Hao, L. Du, H. Wang, W. Xu, X. Zhao, and C. Sun. 2012. Metabonomics analysis of urine and plasma from rats given long-term and low-dose dimethoate by ultra-performance liquid chromatography-mass spectrometry. ChemicoBiological Interactions 199: 143-153.

Fiehn O. 2002. Metabolomics - the link between genotypes and phenotypes. Plant Mol Biol 48: 151-171.

Fukushima A. andM. Kusano. 2013. Recent progress in the development of metabolomedatabases for plant systems biology.Front Plant Sci. 4:73. doi: 10.3389/fpls.2013.00073.

Gu H., Z. Pan, B. Xi, B.E. Hainline, N. Shanaiah, V. Asiago,G.A.N. Gowda, and D. Raftery. 2009. 1H NMR metabolomics study of age profiling in children. NMR In Biomedicine 22: 826-833.

Gunier R.B., M.H. Ward, M. Airola,E.M. Bell, J. Colt, M. Nishioka, P.A. Buffler, P. Reynolds, R.P. Rull, A. Hertz, C. Metayer and J.R. Nuckols. 2011. Determinants of agricultural pesticide concentrations in carpet dust. Environ Health Perspect 119: 970-976. Hao DF, Xu W, Wang H, Du LF, Yang JD, Zhao XJ, Sun C.H. 2012. Metabolomic analysis of the toxic effect of chronic low-dose exposure to acephate on rats using ultra-performance 
liquid chromatography/mass spectrometry. Ecotoxicology and Environmental Safety 83: 2533.

Hebels D.G., P. Georgiadis, H.C. Keun,T.J. Athersuch, P. Vineis, R. Vermeulen, L. Portengen, I.A. Bergdahl, G. Hallmans, D. Palli, B. Bendinelli, V. Krogh, R. Tumino, C. Sacerdote, S. Panico, J.C.S. Kleinjans, T.M.C.M De Kok, M.T. Smith and S.A. Kyrtopoulos. 2013. Performance in omics analyses of blood samples in long-term storage: opportunities for the exploitation of existing biobanks in environmental health research. Environ Health Perspect. 121:480-487.

Holmes E., R.L. Loo, J. Stamler, M. Bictash, I.K. Yap, Q. Chan, T. Ebbels, M. de Iorio, I.J. Brown, K.A. Veselkov, M.L. Daviglus, H. Kesteloot, H. Ueshima, L. Zhao, J.K. Nicholson, and P. Elliott. 2008. Human metabolic phenotype diversity and its association with diet and blood pressure. Nature 453: 396-400.

Idborg H., L. Zamani, P.O. Edlund, I. Schuppe-Koistinen, and S.P. Jacobsson. 2005. Metabolic fingerprinting of rat urine by LC/MS Part 1. Analysis by hydrophilic interaction liquid chromatography-electrospray ionization mass spectrometry. J. Chromatogr. B 828: 913.

Jamin E., N. Bonvallot, M. Tremblay-Franco, J-P. Cravedi, C. Chevrier, S. Cordier, and L. Debrauwer. 2013. Untargeted profiling of pesticide metabolites by LC-HRMS: an exposomics tool for human exposure evaluation. Anal Bioanal Chem. DOI 10.1007/s00216013-7136-2.

Jourdan F., L. Cottret, L. Huc, D. Wildridge, R. Scheltema, H. Hillenweck, M.P. Barrett, D. Zalko, D.G. Watson, and L. Debrauwer. 2010. Use of reconstituted metabolic networks to assist in metabolomic data visualization and mining. Metabolomics 6(2):312-321.

Kanehisa M. and S. Goto. 2000. KEGG: Kyoto Encyclopedia of Genes and Genomes. Nucleic Acids Res 28:27-30. 
Kenny L.C., D. Broadhurst, M. Brown, W.B. Dunn, C.W.G. Redman, D.B. Kell, and P.N. Baker. 2008. Detection and identification of novel metabolomic biomarkers in preeclampsia. Reprod Sci 15: 591-597.

Kholodenko B., M.B. Yaffe, and W. Kolch. 2012. Computational approaches for analyzing information flow in biological networks. Sci Signal 5: 1-14.

Kim K.B., S.H. Kim, S.Y. Um, M.W. Chung, J.S. Oh, S.C. Jung, T.S. Kim, H.J. Moon, S.Y. Han, H.Y. Oh, B.M. Lee, and K.H. Choi. 2009. Metabolomics approach to risk assessment: methoxyclor exposure in rats. J. Toxicol. Environ. Health A Cur. Issues. 72: 1352-1368.

Kim K.B., J.Y. Yang, S.J Kwack, K.L. Park, H.S. Kim, D.H. Ryu, Y.J. Kim, G.S. Hwang and B.M. Lee. 2010. Toxicometabolomics of Urinary Biomarkers for Human Gastric Cancer in a Mouse Model. J. Toxicol. Environ. Health A Cur Issues. 73:1420-1430.

Kochhar S., D.M. Jacobs, Z. Ramadan, F. Berruex, A. Fuerholz, and L.B. Fay. 2006. Probing gender-specific metabolism differences in humans by nuclear magnetic resonance-based metabonomics. Anal Biochem 352: 274-281.

Koek M., R.H. Jellema, J. van der Greef, A.C. Tas, and T. Hankemeier. 2011. Quantitative metabolomics based on gas chromatography mass spectrometry: status and perspectives. Metabolomics 7: 307-328.

Kuo C.H., K.C. Wang, T.F. Tian, M.H. Tsai, Y.M. Chiung, C.M. Hsiech, S.J. Tsai, S.Y. Wang, D.M. Tsai, C.C. Huang, and Y.J. Tseng. 2012. Metabolomic characterization of laborers exposed to welding fumes. Chem Res Toxicol 25: 676-686.

Lacroix M.Z., S. Puel, S.H. Collet, T. Corbel, N. Picard-Hagen, P.L. Toutain, C. Viguié, V. Gayrard. 2011. Simultaneous quantification of bisphenol A and its glucuronide metabolite (BPA-G) in plasma and urine: applicability to toxicokinetic investigations.Talanta85(4):20532059. 
Lenz E.M., J. Bright, I.D. Wilson, A. Hughes, J. Morrisson, H. Lindberg, and A. Lockton. 2004. Metabonomics, dietary influences and cultural differences: a 1H NMR-based study of urine samples obtained from healthy British and Swedish subjects. J Pharm Biomed Anal 36: 841-849.

Li L., R. Li, J. Zhou, A. Zuniga, A.E. Stanislaus, Y. Wu, T. Huan, J. Zhang, Y. Shi, D.S. Wishart and G. Lin. 2013. MyCompoundID: using evidence-based metabolome library for metabolite identification. Anal Chem 85(6):3401-3408.

Liang Y.J., H.P. Wang, L. Yang, W. Li, and Y.J. Wu. 2012a. Metabonomic responses in rat urine following subacute exposure to propoxur. Int J Toxicol 31(3):287-293.

Liang Y.J., H.P. Wang, D.X. Long, and Y.J. Wu. 2012b. (1)H NMR-based metabonomic profiling of rat serum and urine to characterize the subacute effects of carbamate insecticide propoxur. Biomarkers 17: 566-574.

Liang Y.J., Wang H.P., Long D.X., Wu Y.J. 2012c. Applying biofluid metabonomic techniques to analyze the combined subchronic toxicity of propoxur and permethrin in rats. Bioanalysis 4: 2897-2907.

Lindon J.C., E. Holmes, M.E. Bollard, E.G. Stanley, and J.K. Nicholson. 2004. Metabonomics technologies and their applications in physiological monitoring, drug safety assessment and disease diagnosis. Biomarkers 9: 1-31.

Lindon J.C. and J.K. Nicholson. 2008. Analytical techniques for metabonomics and metabolomics, and multi-omic information recovery. TrAC Trends Anal Chem 27: 194-204. Lioy P.J. and S.M. Rappaport. Exposure Science and the Exposome: An Opportunity for Coherence in the Environmental Health Sciences. 2011. Environ Health Perspect 109(11):A466-A467.

Madsen R., T. Lundstedt, and J. Trygg. 2010. Chemometrics in metabolomics - a review in human disease diagnosis. Analytica Chimica Acta 659: 23-33. 
Maher A.D., S.F. Zirah, E. Holmes, and J.K. Nicholson. 2007. Experimental and analytical variation in human urine in $1 \mathrm{H}$ NMR spectroscopy-based metabolic phenotyping studies. Anal Chem 79: 5204-5211.

Mamas M., W. Dunn, L. Neyses, and R. Goodacre. 2011. The role of metabolites and metabolomics in clinically applicable biomarkers of disease. Archives of Toxicology 85: 517.

Merhi M., C. Demur, C. Racaud-Sultan, J. Bertrand, C. Canlet, F.B. Estrada, and L. GametPayrastre. 2010. Gender-linked haematopoietic and metabolic disturbances induced by a pesticide mixture administered at low dose to mice. Toxicology 267: 80-90.

Miller M.G. 2007. Environmental metabolomics: a SWOT analysis (strengths, weaknesses, opportunities, and threats). J Proteome Res 6: 540-545.

Molinska A., L. Blanchet, L.M.C. Buydens, S.S. Wijmenga. 2012. NMR and pattern recognition methods in metabolomics: from data acquisition to biomarker discovery: a review. Anal Chim Acta 750:82-97.

Munoz-Quezada M.T., V. Iglesias, B. Lucero, K. Steenland, D.B. Barr, K. Levy, P.B. Ryan, S. Alvarado and C. Concha. 2012. Predictors of exposure to organophosphate pesticides in schoolchildren in the Province of Talca, Chile. Environ Int 47: 28-36.

Neyraud E., M. Tremblay-Francon, S. Gregoire, O. Berdeaux and C. Canlet. 2013. Relationships between the metabolome and the fatty acidcomposition of human saliva; effects of stimulation. Metabolomics 9:213-222.

Peakman T.C., Elliott P. 2008. The UK Biobank sample handling and storage validation studies. International Journal Of Epidemiology 37 Suppl 1: i2-i6.

Putri S.P., Yamamoto S., Tsugawa H., Fukusaki E. 2013. Current metabolomics: Technological Advances. J Biosci Bioeng. http://dx.doi.org/10.1016/j.jbiosc.2013.01.004 
Ramautar R., Somsen G.W., and de Jong G.J. 2013. CE-MS for metabolomics: developments and applications in the period 2010-2012. Electrophoresis 34: 86-98.

Rappaport S.M. 2011. Implication of the exposome for exposure science. J Expo Sci Environ Epidemiol21(1):5-9.

Robertson D.G., P.B. Watkins, and M.D. Reily. 2011. Metabolomics in Toxicology: Preclinical and Clinical Applications. Toxicol Sci 120: S146-70.

Robertson D. 2005. Metabonomics in toxicology: A review. Toxicol Sci 85: 806-822.

Ross A., G. Schlotterbeck, F. Dieterle, and H. Senn. 2007. NMR spectroscopy techniques for application to metabonomics. In: The handbook of metabonomics and metabolomics. Lindon JC, Nicholson JK, Holmes H (eds):55-112.

Shintu L., R. Baudoin, V. Navratil, J-M. Prot, C. Pointoizeau, M. Defernez, B. Blaise, C. Domange, A.R. Péry, P. Toulhoat, C. Legallais, C. Brochot, E. Leclerc and M-E. Dumas. 2012. Metabolomics-on-a-Chip and Predictive Systems Toxicology inMicrofluidic Bioartificial Organs. Anal. Chem. 84:1840-1848.

Sumner S., Snyder R., Burgess J., Myers C., Tyl R., Sloan C., Fennell T. 2009. Metabolomics in the assessment of chemical-induced reproductive and developmental outcomes using noninvasive biological fluids: application to the study of butylbenzyl phthalate. J Appl Toxicol 29: 703-714.

Sun X., Xu W, Zeng Y, Hou Y, Guo L, Zhao X, Sun C. 2012. Metabonomics evaluation of urine from rats administered with phorate under long-term and low-level exposure by ultraperformance liquid chromatography-mass spectrometry. J Appl Toxicol. doi: 10.1002/jat. 2848

Sun R., J. Zhang, M. Xiong, Y. Chen, L. Yin and Y. Pu. 2012. Metabolomics biomarkers for subacute toxicity screening for benzene exposure in mice. J Toxicol Environ health A 75(18):1163-11-73. 
Sun J., L. Schnackenberg, L. Pence, S. Bhattacharyya, D. Doerge, J. Bowyer and R. Beger. 2010. Metabolomic analysis of urine from rats chronically dosed with acrylamide using NMR and LC/MS. Metabolomics 6(4):550-563.

Theodoridis G.A., H.G. Gika, E.J. Want, and I.D. Wilson. 2012. Liquid chromatography-mass spectrometry based global metabolite profiling: A review. Anal Chim Acta 711: 7-16.

Trygg J. and S. Wold. 2002. Orthogonal projections to latent structures. J Chemometrics 16: 119-128.

Tuplan D, S. Léger, L. Belliveau, A. Culf, and M. Čuperlović-Culf. 2011. MetaboHunter: an automatic approach for identification of metabolites from 1H-NMR spectra of complex mixtures. BMC Bioinfo 12:400.

van den Berg R., Hoefsloot H., Westerhuis J., Smilde A., van der Werf M. 2006. Centering, scaling, and transformations: improving the biological information content of metabolomics data. BMC Genomics 7: 142

van Ravenzwaay B., G. Coelho-Palermo Cunha, V. Strauss, J. Wiemer, E. Leibold, H. Kamp, T. Walk, W. Mellert, R. Looser, A. Prokoudine, E. Fabian, G. Krenrish, and M. Herold. 2010. The individual and combined metabolite profiles (metabolomics) of dibutylphthalate and di(2ethylhexyl)phthalate following a 28-day dietary exposure in rats. Tox Letters 198: 159-170.

van Vliet E, S. Morath, C. Eskes, J. Linge, J. Rappsilber, P. Honegger, T. Hartung and S. Coecke. 2008. A novel in vitro metabolomics approach for neurotoxicity testing, proof of principle for methylmercury, mercury chloride and caffeine. Neurotoxicol 29(1):1-12.

Vineis P., K. van Veldhoven, M. Chadeau-Hyam and T.J. Atersuch. 2013. Advancing the application of omics-based biomarkers in environmental epidemiology. Environ Mol Mutagen. doi: 10.1002/em.21764. 
Vlaanderen J., L.E. Moore, M.T. Smith, Q. Lan, L. Zhang, C.F. Skibola, N. Rothman, and R. Vermeulen. 2010. Application of OMICS technologies in occupational and environmental health research; current status and projections. Occup Environ Med 67: 136-143.

Vulimiri S.V., M. Misra, J.T. Hamm, M. Mitchell, and A. Berger. 2009. Effects of mainstream cigarette smoke on the global metabolome of human lung epithelial cells. Chem Res Toxicol 22(3):492-503.

Wang H.P., Y.J. Liang, D.X. Long, J.X. Chen, W.Y. Hou, and Y.J. Wu. 2009. Metabolic profiles of serum from rats after subchronic exposutre to chlorpyrifos and carbaryl. Chem Res Toxicol 22(6):1026-1033.

Wang H.P., Y.J. Liang, Q. Zhang, D.X. Long, W. Li, L. Li, L. Yang, X.Z. Yan, and Y.J. Wu. 2011. Changes in metabolic profiles of urine from rats following chronic exposure to anticholinesterase pesticides. Pestic Biochem Physiol 101(3):232-239.

Wang H.P., Y.J. Liang, Y.J. Sun, J.X. Chen, W.Y. Hou, D.X. Long, and Y.J. Wu. 2013. 1H NMR-based metabonomic analysis of the serum and urine of rats following subchronic exposure to dichlorvos, deltamethrin, or a combination of these two pesticides. ChemicoBiological Interactions 203: 588-596.

Waterman D.S., F.W. Bonner, and J.C. Lindon. 2009. Spectroscopic and statistical methods in metabonomics. Bioanalysis 1: 1559-1578.

Werner E., J.F. Heilier, C. Ducruix, E. Ezan, C. Junot, and J.C. Tabet. 2008. Mass spectrometry for the identification of the discriminating signals from metabolomics: current status and future trends. J Chromatogr B 871: 143-163.

Wild C.P. 2005. Complementing the genome with an "exposome": The outstanding challenge of environmental exposure measurement in molecular epidemiology. CEBP 14: 1847-1850. Wishart D.S., D. Tzur,C. Knox, R. Eisner, A.C. Guo, N. Young, D. Cheng, K. Jewell, D. Arndt, S. Sawhney, C. Fung, L. Nikolai, M. Lewis, M-A. Coutouly, I. Forsythe, P. Tang, S. 
Shrivastava, K. Jeroncic, P. Stothard, G. Amegbey, D. Block, D.D. Hau, J. Wagner, J. Miniaci, M. Clements, M. Gebremedhin, N. Guo, Y. Zhang, G.E. Duggan, G.D. MacInnis, A.M. Weljie, R. Dowlatabadi, F. Bamforth, D. Clive, R. Greiner, L. Li, T. Marrie, B.D. Sykes, H.J. Vogel, and L. Querengesser. 2007. HMDB: The human metabolome database. Nucleic Acids Res 35: D521-D526.

Wishart D.S., T. Jewison, A.C. Guo, M. Wilson, C. Knox, Y. Liu, Y. Djoumbou, R. Mandal, F. Aziat, E., S. Bouatra, I. Sinelnikov, D. Arndt, J. Xia, P. Liu, F. Yallou, T. Bjorndahl, R. Perez-Pineiro, R. Eisner, F. Allen, V. Neveu, R. Greiner, andA. Scalbert. 2013. HMDB 3.0 The Human Metabolome Database in 2013.Nucleic Acids Res. 41:D801-7. doi: 10.1093/nar/gks1065.

Wishart D.S., C. Knox, A.C.Guo, R. Eisner, N. Young, B. Gautam, D.D. Hau, N. Psychogios, E. Dong, S. Bouatra, R. Mandal, I. Sinelnikov, J; Xia, L. Jia, J.A. Cruz, E. Lim, C.A. Sobsey, S. Shrivastava, P. Huang, P. Liu, L. Fang, J. Peng, R. Fradette, D. Cheng, D. Tzur, M. Clements, A. Lewis, A. De Souza, A. Zuniga, M. Dawe, Y. Xiong, D. Clive, R. Greiner, A. Nazyrova, R. Shaykhutdinov, L. Li, H.J. Vogel and I. Forsythe. 2009. HMDB: a knowledgebase for the human metabolome. Nucleic Acids Res. 37: D603-D610. doi: 10.1093/nar/gkn810.

Wold S., M. Sjostrom, and L. Eriksson. 2001. PLS-regression: a basic tool of chemometrics. Chem Intel Lab Syst 58: 109-130.

Xia H.F., Y. Chi, X. Qi, M.M. Su, Y. Cao, P.P. Song, X. Li, T.L. Chen, A.H. Zhao, Y.N. Zhang, Y. Cao, X. Ma, and W. Jia. 2011. Metabolomic evaluation of di-n-butyl phthalateinduced teratogenesis in mice. Metabolomics 7(4):559-571.

Zhang A., H. Sun and X. Wang. 2012. Saliva metabolomics opens door to biomarker discovery, disease diagnosis, and treatment.Appl Biochem Biotechnol. 168(6):1718-1727. 
Xu Y., S. Agrawal, T.J. Cook, and G.T. Knipp. 2007. Di-(2-ethylhexyl)-phthalate affects lipid profiling in fetal rat brain upon maternal exposure. Arch Toxicol 81(1):57-62.

Yang J., X. Sun, Z. Feng, D. Hao, M. Wang, X. Zhao, and C. Sun. 2011. Metabolomic analysis of the toxic effects of chronic exposure to low-level dichlorvos on rats using ultraperformance liquid chromatography-mass spectrometry. Toxicol Letters 206: 306-313.

Yang J. H Wang, W. Xu, D. Hao, L. Du, X. Zhao and C. Sun. 2013. Metabolomic analysis of rat plasma following chronic low-dose exposure to dichlorvos. Hum Exp Toxicol 32(2):196205.

Zhang H., L. Ding, X. Fang, Z. Shi,Y. Zhang, H. Chen, X. Yan, and J. Dai. 2011. Biological responses to perfluorododecanoic acid exposure in rat kidneys as determined by integrated proteomic and metabonomic studies. PLoS ONE 6: e20862-1-11.

Zhang Y., B. Wu, Z.Y. Zhang, and S.P. Cheng. 2011. A metabonomic analysis on health effects of drinking water on male mice (Mus musculus). J Hazard Mat 190: 515-519.

Zhang J., L. Yan, M. Tian, Q. Huang, S. Peng, S. Dong, and H. Shen. 2012. The metabonomics of combined dietary exposure to phthalates and polychlorinated biphenyls in mice. J Pharm Biomed Anal 66: 287-297.

Zhou B, J. Wang, and H.W. Ressom. 2012. MetaboSearch: tool for mass-based metabolite identification using multiple databases. PolsOne 7(6):e40096.

Zivkovic A., M. Wiest, U. Nguyen, R. Davis, S. Watkins, andJ. German. 2009. Effects of sample handling and storage on quantitative lipid analysis in human serum. Metabolomics 5: 507-516. 


\section{Figure Legends}

Figure 1: Diagram of the toxicity of a contaminant and the link with the metabolic fingerprint

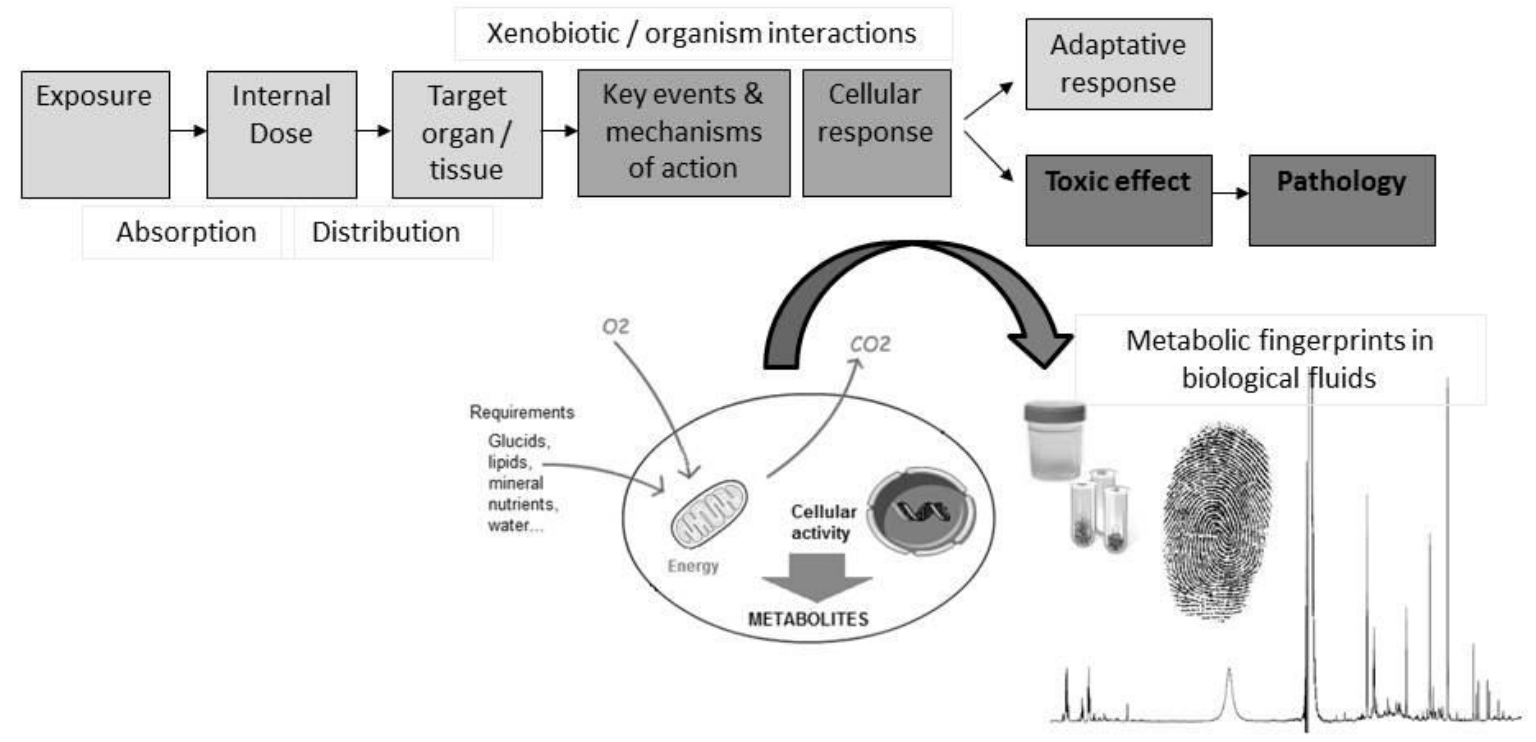


Figure 2: flowchart of a metabolomics analyses

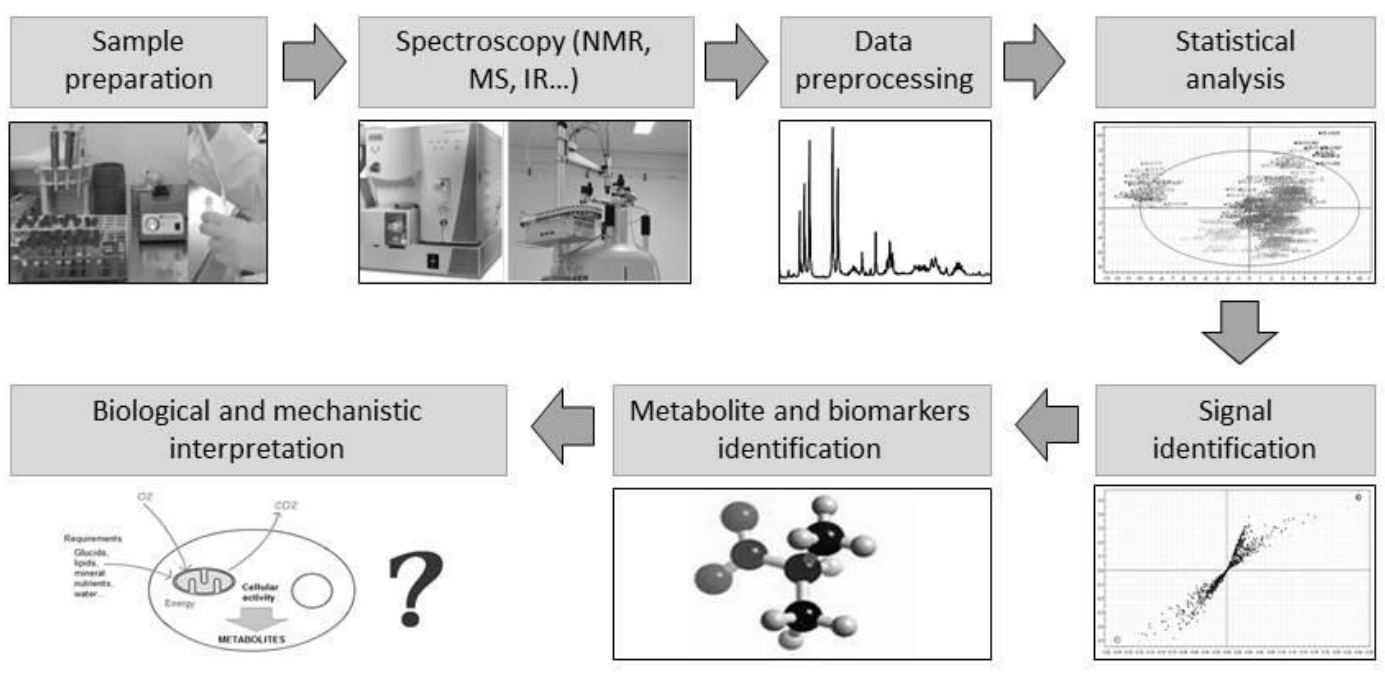

